\title{
La préverbation en français médiéval : polysémie et sens grammatical
}

\author{
Monique Dufresne \\ Université Queen's \\ dufresne@queensu.ca \\ Fernande Dupuis \\ dupuis.fernande@uqam.ca \\ Université du Québec à Montréal \\ Mireille Tremblay \\ mt11@queensu.ca
}

\section{Introduction}

La plupart des grammairiens tiennent pour acquis que les items lexicaux sont organisés en deux types de classes de mots : aux catégories dites lexicales comme nom, adjectif, verbe et adverbe s'opposent les catégories grammaticales telles déterminant, complémenteur, marques casuelles et aspectuelles, verbes auxiliaires, etc. Parmi les critères permettant de distinguer les unités grammaticales des unités lexicales, Abney 1987 cite la taille de l'inventaire (clos vs. Illimité), la dépendance phonologique, le nombre de compléments, la séparabilité et le contenu sémantique (mots-outils vs. mots sémantiquement pleins).

Par ailleurs, il est aussi souvent admis que cette division n'est pas catégorique et qu'il existe, d'une part, des catégories dites semi-lexicales (Corver \& Van Riemsdijk 2001) telles les prépositions, les verbes supports, les adjectifs de quantifications, les particules partageant les caractéristiques des deux classes et, d'autre part, un nombre considérable d'unités lexicales oscillant entre les deux pôles. Comme le remarque si bien Victorri (1999), «l'un des problèmes essentiels provient de l'abondance des unités polycatégorielles [...] Doit-on considérer qu'il y autant de catégories homonymiques que de catégories syntaxiques possibles pour ces termes, dans l'espoir de préserver une dichotomie entre unités grammaticales et lexicales ? "Si l'étude de la polycatégorialité s'est souvent limitée aux items lexicaux syntaxiquement autonomes (particules, verbes supports, quantificateurs et classificateurs) et peu de recherches ont considéré la question de la polycatégorialité des items lexicaux non autonomes.

Dans ce qui suit, nous chercherons à combler ce vide en nous penchant sur un cas de polycatégorialité dans le domaine morphologique : le système préfixal de l'ancien français. Ce système, d'origine prépositionnelle (Nyrop 1956, Buridant 1995, Wilmet 1998), peut prendre une multitude de valeurs, surtout directionnelles et aspectuelles, mais aussi réciproques. Notre étude sera organisée de la façon suivante. La première section porte principalement sur les problèmes soulevés par la polysémie et l'apparente polycatégorialité des préfixes en ancien français. Dans la deuxième section, nous présentons une description exhaustive des propriétés sémantiques et morphosyntaxiques de quatre préfixes représentatifs en français médiéval (re-, entre-, sor- et $a$ - et faisons part de la méthodologie utilisée pour distinguer la préfixation productive de la préfixation non productive. Nous montrerons l'ensemble des stratégies informatiques que nous avons développées à l'aide du logiciel SATO (sous la forme de fichiers exécutables d'ensemble d'expressions régulières, lemmatisation, dictionnaires, parsage...) pour cerner l'évolution du phénomène de la préverbation. La troisième section présente une analyse unifiée des préfixes re-, entre- et $a$-. Nous montrons comment les interprétations directionnelle et aspectuelle, ainsi que l'interprétation réciproque des préfixes peuvent découler du sémantisme des prépositions, sans avoir recours à des mécanismes aussi coûteux que la grammaticalisation. Finalement, dans la dernière section, nous montrons comment notre analyse permet une meilleure compréhension de la distinction lexicalgrammatical. Nos données sont tirées de la Base de français médiéval, élaborée par Christiane Marchello- 
Nizia (ENS, LYON), du Dictionnaire Larousse de l'ancien français et du Tobler-Lommatzsch Altfranzösisches Wörterbuch.

\section{La préverbation en français médiéval}

\subsection{Aperçu du système préfixal}

L'ancien français est caractérisé par un système aspectuel dérivationnel. Comme le montrent les exemples ci-dessous, de nombreux préfixes servent à modifier la valeur aspectuelle des verbes (l'aktionsart, c'est-àdire la façon dont on perçoit la structure temporelle interne d'une situation (Comrie 1976)).

$\begin{array}{llll}\text { A : } & \text { river « longer la rive } & \text { a-river } & \text { «toucher la rive » } \\ \text { Sur : } & \text { saillir } & \text { sor-saillir } & \text { « sauter » } \\ \text { De : } & \text { parler } & \text { de-parler } & \text { «parler en mal de» } \\ \text { Pour : } & \text { tenir } & \text { por-tenir } & \text { «posséder » } \\ \text { Par : } & \text { fermer } & \text { par-fermer } & \text { «ratifier » }\end{array}$

Plusieurs arguments montrent que les verbes préfixés de l'AF sont formés dans le composant morphologique. D'abord, la préfixation affecte la structure d'argument et les propriétés casuelles du prédicat. Il a déjà été montré que l'ajout du préfixe $a$-rend quasi obligatoire la présence d'un argument locatif (38\% versus 81\%). Par ailleurs, le sens du verbe préfixé n'est pas toujours compositionnel. Ainsi, abaiser avait deux sens : un sens compositionnel (« faire descendre à un niveau plus bas ») et un sens non compositionnel («apaiser, éteindre»). Les glissements sémantiques sont caractéristiques des objets morphologiques, qui sont plus souvent lexicalisés. De plus, le verbe préfixé semble pouvoir servir de base à la dérivation, les infinitifs préfixés étant souvent nominalisés (2).

(2) Au departir li redemande, / La belle Yseut, anuit viande 'Au départir lui redemande / La belle Yseut journée nourriture' «Au moment du partage, la belle Yseut lui redemande des provisions pour la journée...»

Béroul, p. 121

Finalement, le verbe préfixé forme une unité lors du déplacement du verbe (l'AF était une langue V2).

(3) Ademplir voeill vostre cumandement.

«Je veux accomplir vos ordres. »

Roland, XXII, 309

\subsection{Problématique}

La plupart de ces préfixes sont polysémiques et peuvent prendre un sens soit directionnel, soit aspectuel. Par exemple, le préfixe en- peut indiquer soit le mouvement vers l'intérieur (4a) ou entourant un certain espace (4b), soit l'inchoatif (4c). De même, le préfixe re-peut signifier le retour à une position antérieure (5a), le recommencement d'un procès $(5 \mathrm{~b})$, ou l'intensivité d'un procès $(5 \mathrm{c})$, alors que le préfixe sorajoute l'idée de dépassement de supériorité (6a) ou de démesure (6b).

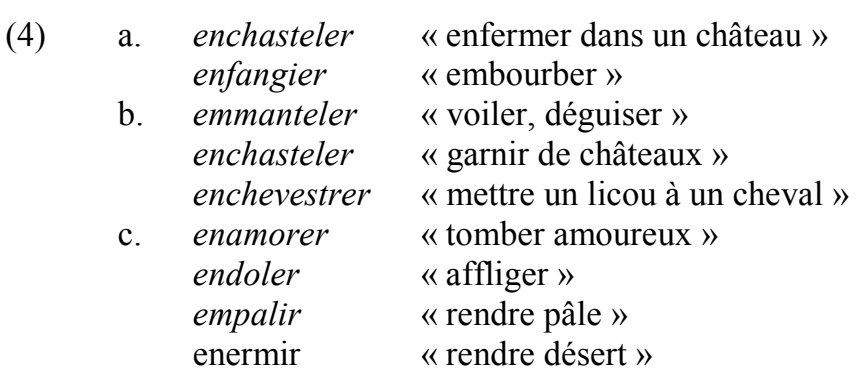



a. revenir
retourner
b. refonder
regendrer
c. refuir
regarder
regoloser

(6) a. soraler

b. $\quad \begin{aligned} & \text { surboivre } \\ & \text { soreissir } \\ & \text { soratendre }\end{aligned}$

«passer par dessus »
«boire démesurément "
" déborder»
« attendre trop, en vain»

(exemples tirés du Dictionnaire de l'ancien français)

Parmi les préfixes aspectuels, il en est un toutefois qui semble avoir un comportement plus hétéroclite: le préfixe entre-. En effet, ce préfixe polysémique prend, en plus d'une valeur aspectuelle (procès accompli à moitié) comme dans entreclore, une valeur non pas directionnelle mais réciproque comme dans s'entrevenir, s'entreplevir.
a. entrecorre
« courir à travers »
entraler
« s'interposer»
entreclore
« entrouvrir»
entregeter
« intercaler »
b. s'entraler
« aller l'un vers l'autre »
s'entramer
« s'aimer l'un l'autre»
s'entreplevir
« s'engager mutuellement»
s'entrevenir
« aller l'un contre l'autre, se rencontrer »

Comment rendre compte de la polysémie des préfixes) ? Comment expliquer la synonymie de nombreux préfixes, comme l'attribution du sens intensif aux préfixes $a-$, con-, en-, re- ? Finalement, comment expliquer qu'occasionnellement, ces mêmes préfixes ne semblent avoir aucun apport sémantique comme le montrent les exemples en (8)?
a. conter, aconter
b. conchier, enconchier
cheoir, encheoir
cliner, encliner
c. tourner ariere, retourner ariere
« compter, conter, raconter »
« souiller»,
« tomber»
« incliner, pencher »
« retourner en arrière »

A ces questions sémantiques s'ajoutent des questions d'ordre syntaxique. Quelle est la relation entre prépositions spatiales, préfixes directionnels et préfixes aspectuels) ? Les préfixes aspectuels de l'ancien français sont-ils le résultat d'un processus de grammaticalisation, processus qui aurait permis le changement de préfixes de catégorie Préposition en préfixes de catégorie Aspect. L'autre possibilité, la polysémie, est confrontée à deux défis. D'une part, il faut montrer que l'ensemble des sens associés à une entrée lexicale donnée n'est pas arbitraire. D'autre part, il faut rendre compte de l'apparente polycatégorialité des formes sans avoir recours à la grammaticalisation ou à la conversion (qui réduiraient toutes deux la polycatégorialité à l'homonymie). Si la distinction grammatical-lexical est une propriété binaire (plutôt qu'un continuum), les cas de polycatégorialité devraient pouvoir être réduits soit à des items purement lexicaux, soit à des items purement grammaticaux. 


\section{Méthodologie}

\subsection{Constitution du corpus}

Notre étude se limite à l'étude systématique de quatre préfixes de l'ancien français : les préfixes re-, entre-, $a$ - et sor-. Ces préfixes manifestent des statuts morphosyntaxiques distincts : alors que $a$ - et sordoivent obligatoirement être attachés à une racine verbale, re- et entre- exhibent une plus grande liberté et peuvent apparaître séparés de la racine verbale par un pronom ou un auxiliaire (9).

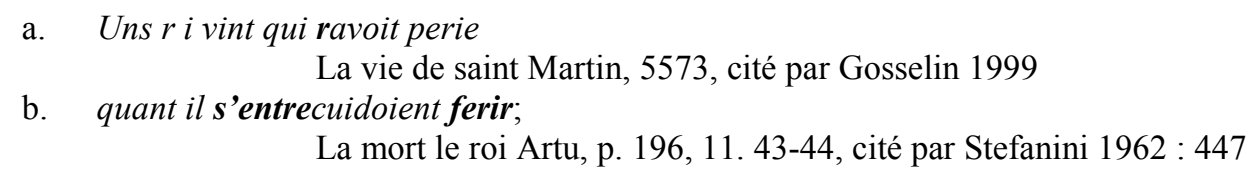

La liste des formes préfixées a été établie de façon automatique à l'aide du logiciel d'analyse de textes SATO élaboré par François Daoust, UQAM. Afin de distinguer les verbes préfixés comme ariver (a+river) des verbes non préfixés comme aller, nous avons utilisé une procédure automatique d'identification des verbes préfixés, suivie d'une vérification systématique de la liste des verbes préfixés obtenus dans les dictionnaires (Tobler-Lommatzsch, Dictionnaire Larousse de l'ancien français,). Nous avons choisi d'exclure les verbes dénominaux (comme ataverner "s'atabler à une taverne " et désadjectivaux (comme embelir) et les formes à double préfixe comme renchoir "rendre amoureux », enamorer « retomber, récidiver».

\subsection{Identification des valeurs productives des préfixes}

Nous avons associé à chacun des quatre préfixes les valeurs sémantiques suivantes :

- le préfixe $a$ - a été codé pour les valeurs Terminatif, Inchoatif et Intensif;

- le préfixe entre- pour les valeurs Réciproque (s'entreamer "s'aimer l'un l'autre »), Locative (entremêler) et Autres formes (entreprendre, entremetre);

- le préfixe re- a été codé pour les valeurs Directionnel (Retorner), Iteratif (redemander), Intensif (regarder), Pronominal (se recorder) et Autres formes (recroire) ;

- le préfixe sor- a été codé pour seulement deux valeurs : Locatif (soraler "passer par dessus », soreissir " déborder ») et Superlatif (surboivre "boire démesurément » et soratendre "attendre trop, en vain »)

L'analyse des résultats de nos premières codifications des corpus de textes confirme le caractère productif de la préfixation verbale en ancien français.

Tableau 1a: Préfixation en A- dans le corpus 13prose

\begin{tabular}{|l|l|l|}
\hline & Nombre d'occurrences & $\%$ \\
\hline Terminatif/locatif & 2067 & $92,11 \%$ \\
\hline Inchoatif & 158 & $7,04 \%$ \\
\hline Intensif & 19 & $0,85 \%$ \\
\hline Total & 137 & $100 \%$ \\
\hline
\end{tabular}

Tableau 1b : Préfixation en ENTRE- dans le corpus 13prose

\begin{tabular}{|l|l|l|}
\hline & Nombre d'occurrences & $\%$ \\
\hline Locatif (entremêler) & 2 & $1,5 \%$ \\
\hline Réciproque & 101 & $73,7 \%$ \\
\hline
\end{tabular}




\begin{tabular}{|l|l|l|}
\hline Différent & 34 & $24,8 \%$ \\
\hline Total & 137 & $100 \%$ \\
\hline
\end{tabular}

Tableau 1c: Préfixation en RE- dans le corpus 13prose

\begin{tabular}{|l|l|l|}
\hline & Nombre d'occurrences & $\%$ \\
\hline Locatif & 661 & $45,46 \%$ \\
\hline Intensif & 611 & $42,02 \%$ \\
\hline Itératif & 172 & $11,83 \%$ \\
\hline Pronominal & 4 & $0.57 \%$ \\
\hline Différent & 4 & $0.57 \%$ \\
\hline
\end{tabular}

Tableau 1d : Préfixation en SOR- dans le corpus 13 prose

\begin{tabular}{|l|l|l|}
\hline & Nombre d'occurrences & $\%$ \\
\hline Locatif & 0 & $0 \%$ \\
\hline Superlatif & 1 & $5,6 \%$ \\
\hline Différent & 17 & $94,4 \%$ \\
\hline Total & 18 & $100 \%$ \\
\hline
\end{tabular}

Ces premières codifications montrent toutefois que les préfixes verbaux ne forment pas une classe homogène, l'hétérogénéité des préfixes se reflétant dans leur productivité. En effet, contrairement aux polycatégories syntaxiquement autonomes comme les verbes supports et les quantificateurs, les polycatégories non autonomes incluent des formes lexicalisées. Ainsi, comme la préfixation verbale était déjà productive en latin, les formes préfixées de l'ancien français peuvent être parvenues dans la langue déjà lexicalisées. Nous avons tenté d'exclure ces formes lexicalisées antérieurement afin d'éliminer les cas de dérive sémantique et d'archaïsmes sémantiques.

La préfixation non productive a été identifiée à partir des deux critères suivants : sens non compositionnel et nombre restreint de formes distinctes. Les formes suivantes ont ainsi été considérées comme lexicalisées : 
Tableau 2a : Formes préfixées en A- lexicalisées dans le corpus 13 prose

\begin{tabular}{|l|l|l|}
\hline & Nombre d'occurrences & $\%$ \\
\hline Aaisier & 4 & $21,5 \%$ \\
\hline Aamer & 3 & $15,79 \%$ \\
\hline Aconter & 10 & $52,63 \%$ \\
\hline Aemplir & 2 & $10,53 \%$ \\
\hline Total & 19 & $100 \%$ \\
\hline
\end{tabular}

Tableau $2 b$ : Formes préfixées en ENTRE- lexicalisées dans le corpus 13 prose

\begin{tabular}{|l|l|l|}
\hline & Nombre d'occurrences & $\%$ \\
\hline S'entremetre & 26 & $76,5 \%$ \\
\hline Entreprendre & 8 & $23,5 \%$ \\
\hline Total & 34 & $100 \%$ \\
\hline
\end{tabular}

Tableau 2c : Formes préfixées en RE- lexicalisées dans le corpus 13prose

\begin{tabular}{|l|l|l|}
\hline & Nombre d'occurrences & $\%$ \\
\hline Recorder & 4 & $50 \%$ \\
\hline Recroire & 4 & $50 \%$ \\
\hline Total & 8 & $100 \%$ \\
\hline
\end{tabular}

Tableau 2d : Formes préfixées en SOR- lexicalisées dans le corpus 13prose

\begin{tabular}{|l|l|l|}
\hline & Nombre d'occurrences & $\%$ \\
\hline Sorprendre & 12 & $70,6 \%$ \\
\hline Sorvenir & 3 & $17,6 \%$ \\
\hline Sorseoir & 1 & $5,9 \%$ \\
\hline Sordre & 1 & $5,9 \%$ \\
\hline Total & 17 & $100 \%$ \\
\hline
\end{tabular}

L'identification des formes lexicalisées nous a permis d'exclure certaines des valeurs associées aux préfixes : nous avons ainsi pu exclure l'interprétation intensive du préfixe $a$-, ainsi que les pourcentages codés Autres formes de tous les préfixes. De plus, notre méthodologie nous a permis de constater que le préfixe sor- n'était déjà plus productif en ancien français.

L'élagage des formes lexicalisées nous a permis d'identifier les valeurs productives des préfixes et donc de cerner les cas de polycatégorialité.

Tableau 3a : Préfixation productive en Adans le corpus de 13 prose 


\begin{tabular}{|l|l|l|}
\hline & Nombre d'occurrences & $\%$ \\
\hline Terminatif/Locatif & 2067 & $92,9 \%$ \\
\hline Inchoatif & 158 & $7,1 \%$ \\
\hline Total & 2225 & $100 \%$ \\
\hline
\end{tabular}

Tableau $3 \mathrm{~b}$ : Préfixation productive en ENTREdans le corpus de 13 prose

\begin{tabular}{|l|l|l|}
\hline & Nombre d'occurrences & $\%$ \\
\hline Locatif & 2 & $1,9 \%$ \\
\hline Réciproque & 101 & $98,1 \%$ \\
\hline Total & 103 & $100 \%$ \\
\hline
\end{tabular}

Tableau 3c : Préfixation productive en RE- dans le corpus de 13 prose

\begin{tabular}{|l|l|l|}
\hline & Nombre d'occurrences & $\%$ \\
\hline Locatif & 661 & $45,78 \%$ \\
\hline Intensif & 611 & $42,3 \%$ \\
\hline Itératif & 172 & $11,9 \%$ \\
\hline Total & 1444 & $100 \%$ \\
\hline
\end{tabular}

\section{Le sémantisme des préverbes : étude qualitative}

Les préfixes de l'ancien français étant d'origine prépositionnelle, deux hypothèses s'offrent à nous pour rendre compte de la relation entre préposition et préfixe. La première hypothèse voudrait qu'il existe une homophonie systématique dans le système prépositionnel de l'ancien français. Ainsi, prépositions et préfixes auraient des entrées lexicales distinctes et la relation entre les formes serait le résultat d'un processus de grammaticalisation, accompagné ou non de changements catégoriel et sémantique. La seconde hypothèse poserait au contraire qu'il n'existe pas de distinctions syntaxiques ou sémantiques inhérentes entre préposition et préfixe. Dans ce cas, la polysémie des préfixes ne serait qu'apparente et les distinctions entre préposition et préfixe ne seraient pas inscrites dans l'entrée lexicale des items lexicaux, mais dériveraient plutôt du contexte morphosyntaxique. Les pages qui suivent militent en faveur de cette dernière hypothèse.

\subsection{Le préfixe entre-}

Nous avons récemment proposé une analyse unifiée du préfixe-préposition entre(-). Cette analyse pose que le préfixe partage le sémantisme de la préposition qui lexicalise la fonction de PARTITION. Cette fonction permet de diviser un ensemble en sous-ensembles.

$$
\text { PART }(\mathrm{x}) \rightarrow \operatorname{PART}\left(\mathrm{x}_{1}+\mathrm{x}_{2} \ldots+\mathrm{x}_{\mathrm{n}}\right)
$$

Pour être divisible en sous-ensembles, l'objet de la préposition doit être une variable plurielle. Cette contrainte de pluralité s'observe non seulement lorsque entre est utilisé comme préposition (il est bien connu que l'objet de la préposition entre doit nécessairement être pluriel), mais aussi dans l'utilisation comme préfixe. 
La préfixation aspectuelle est limitée aux prédicats événementiels comme (11) et semble incompatible avec les états.

\title{
(11) Je regarday jusques a tant/Que une dame d'honneur saysie /M'entrevit,...
}

\author{
Le Roman de la rose
}

Nous attribuons cette différence au contraste aspectuel distinguant les événements des états. Contrairement aux événements, les états sont temporellement homogènes : ils n'ont pas de structure interne et sont indécomposables. En ce sens, ils se comportent comme des noms de masse $e^{\mathrm{i}}$ et sont donc incompatibles avec la fonction de PARTITION. En revanche, les prédicats événementiels sont décomposables en sous-événements (début, milieu et fin) et peuvent donc être quantifiés. Étant fondamentalement pluriels, les prédicats événementiels sont donc compatibles avec le préfixe entre-. En modifiant la fin de l'événement, entre- permet l'interprétation selon laquelle seule une partie de l'événement a été complétée, d'où l'interprétation de procès accompli à moitié.

Contrairement aux constructions aspectuelles, les constructions réciproques impliquant le préfixe entrene sont pas limitées aux prédicats événementiels (12).

$$
\begin{aligned}
& \text {...quant li dui frere s'entrevirent, assez } i \text { ot espandu pleurs et lermes d'une part et d'autre, car } \\
& \text { moult s'entramoient de bone amour, et dist Hestor a Lancelot: }
\end{aligned}
$$

Artu, p. 260

Les verbes réciproques sont essentiellement des prédicats qui impliquent des paires d'individus, créant soit des paires d'événements ou des paires d'état. Par conséquent, les états réciproques sont en fait une pluralité d'états, d'où leur compatibilité avec la fonction de PARTITION qui exige une variable plurielle comme objet.

En résumé, nous avons proposé une analyse selon laquelle la préposition-préfixe entre(-) lexicalise la fonction de PARTITION. Cette analyse permet de rendre compte des interprétations réciproque et aspectuelle du préfixe entre- sans avoir recours à des mécanismes aussi coûteux que la grammaticalisation. La différence d'interprétation de la préposition-préfixe dépend de la nature de la variable: lorsque la variable est un individu, la prédication avec entre(-) construit un groupe d'individus partitionné en sous-ensembles (préposition entre) et lorsque la variable est un événement ou un état réciproque, entre- peut soit partitionner l'événement en sous-événements (entre- aspectuel), soit contribuer à partitionner l'événement ou l'état en paires distribuées d'événements ou d'états (entreréciproque).

\subsection{Le préfixe re-}

A priori, l'approche proposée ci-dessus semble contredite par le préfixe $r e$-, car ce préfixe diffère des autres en ce qu'il n'offre qu'une utilisation préfixale. Ce fait ne devrait toutefois pas nous arrêter puisque le préfixe re- a aussi un correspondant prépositionnel - la particule-préposition ariere - qui apparaît souvent avec le préfixe $r e$ - sans sembler provoquer de changement de sens.

\author{
a. A Vivien est retornez arrier. \\ 'Il retourne auprès de Vivien.' \\ Aliscans : 1073; cité dans Buridant 2000, p. 546 \\ b. Li cuens Guillelmes soz l'arbre retorna \\ 'Le comte Guillaume est revenu sous l'arbre' \\ Aliscans : 1075; cité dans Buridant 2000, p. 546 \\ c. Mes une gent m'a hui fait reculer/ Par grant effroi et arriere torner \\ 'Mais une troupe ennemie m'a fait reculer et tourner bride en proie à un terrible effroi'
}

Aliscans : 991-992; cité dans Buridant 2000, p. 546

Par ailleurs, tout comme le préfixe $r e$-, la particule ariere peut prendre un sens itératif et un sens directionnel. Or, une étude récente a montré qu'en ancien français, les particules ne sont pas marquées 
pour la distinction directionnelle/aspectuelle dans le lexique et que les particules directionnelles et aspectuelles n'ont pas d'entrées lexicales distinctes puisque ces deux interprétations sont en distribution complémentaire: la particule ariere employée avec les verbes qui prennent un argument locatif (14a) (incluant les verbes de mouvement) a une interprétation directionnelle, alors que la particule ariere utilisée avec les autres verbes (14b) a une interprétation aspectuelle.

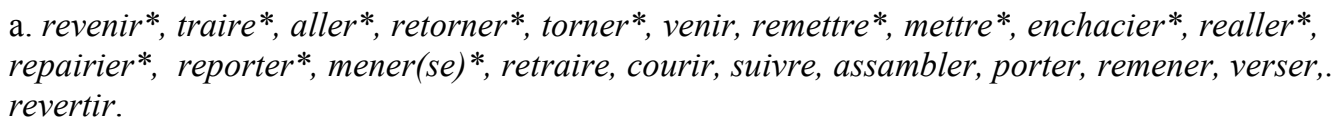

b. consiller*, chier*, bouter, mander, remander, refuser, rebouter, s'abattre, armer

Dans notre communication, nous montrerons que l'interprétation du préfixe re- est aussi dépendante du contexte d'insertion et donc qu'il est possible d'offrir une analyse unifiée du préfixe re-.

\subsection{Le préfixe a-}

Le comportement préfixe d'origine prépositionnelle $a$ - dont le sens premier est direction appuie l'hypothèse que nous proposons. Il modifie la valeur d'un verbe soit en y ajoutant une valeur terminative ou une valeur inchoative. Rappelons que la valeur terminative est de loin la plus productive. Il est important de souligner que cette modification fait en sorte qu'un verbe passe d'imperfectif à perfectif. Ainsi un verbe qui exprime une activité devient un achèvement ou un accomplissement (terminologie que non empruntons à Vendler (1967)), tel que l'illustrent les exemples suivants :

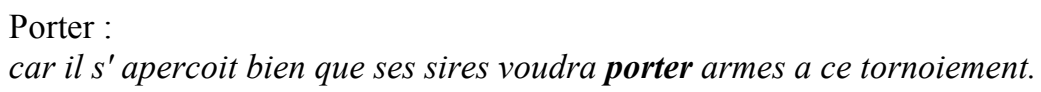

Porter:

car il s' apercoit bien que ses sires voudra porter armes a ce tornoiement.

Artu, 4

(16) Aporter :

Lors demande ses armes, et len li aporte maintenant

$$
\text { Qgraal, } 94
$$

Il est aussi à noter que le préfixe $a$ - ne se retrouve pas avec des procès duratif ou être associé à un procès qui présente déjà une fin inhérente:

*Ils ont aconstruit le castel.

Pour rendre compte du comportement de ce préfixe, posons la structure (18) qui s'inspire du modèle de Di Sciullo (1996, 1999); ce dernier contient dans son sémantisme la valeur positive du trait TERMINATIF. Ainsi lorsque le préfixe s'adjoint à une base verbale sans point de terminaison, il lui en procure une. C'est ce que la structure suivante illustre pour le verbe apporter :

$$
\begin{gathered}
\text { apporter } \\
\mathrm{V}[+\mathrm{S}+\mathrm{T}] \\
/ \backslash \\
\mathrm{P} \mathrm{V} \\
\mathrm{A}[+\mathrm{T}] \quad \text { porter }[+\mathrm{S}, \mathrm{T}]
\end{gathered}
$$

Porter est un verbe duratif porteur du trait $[+\mathrm{S}]$ (selon la terminologie de Di Sciullo) non spécifié pour le trait terminatif [T]. Le préfixe aspectuel $a$ - ajoute un point de terminaison au procès. Ainsi le verbe apporter acquiert le trait [+T]. Comment rendre compte des différences sémantiques qui résultent de l'ajout du préfixe $a$-, puisque tantôt il en résulte un procès terminatif, tantôt un procès inchoatif ? Les faits relevés dans notre corpus nous permettent de poser que si le préfixe s'adjoint à un verbe de mouvement/déplacement son permet au trait [T] de réaliser le point de terminaison. Par ailleurs si le verbe n'exprime pas un mouvement, il en résulte un procès inchoatif, et la valeur aspectuelle est alors dérivée : 
(19) II. homes de blans dras vestus Qui unt Anna si aparlée

« Deux hommes vêtus de draps blancs qui ont adressé la parole à Anna. »

Wace, Marie 28, Tobler, p. 427, tome 1

En terminant, soulignons que la particule-préposition avant présente un comportement similaire à celui de ariere. Ainsi si elle s'associe à un verbe qui ne marque pas le mouvement, le composé verbal qui en résulte a une valeur aspectuelle, soit inchoative, et non une valeur directionnelle: parler avant « commencer à parler ».

\section{Conclusion}

Nous avons montré qu'il est possible de rendre compte de la polycatégorialité des préfixes de l'ancien français sans avoir recours à la grammaticalisation ou à la conversion (qui réduiraient toutes deux la polycatégorialité à l'homonymie). Or, si la distinction grammatical-lexical est une propriété binaire (plutôt qu'un continuum), les cas de polycatégorialité devraient pouvoir être réduits soit à des items purement lexicaux, soit à des items purement grammaticaux.

En proposant une analyse unifiée des préfixes et des prépositions, nous avons montré que les effets de polycatégorialité sont une autre propriété permettant de distinguer les catégories lexicales des catégories grammaticales.

\section{Références bibliographiques}

Abney, S. (1987) The English NP in its Sentential Aspect. Thèse de doctorat, MIT, Cambridge, MA

Buridant, C. (1995) «Les préverbes en ancien français», in A. Rousseau (ed.) Les préverbes dans les langues Europe : introduction à l'étude de la préverbation, Presses du Septentrion, Université de Lille III

Buridant, C. (2000) Grammaire nouvelle de l'ancien français. Paris: Sedes.

Comrie, Bernard. 1976. Aspect. Cambridge: Cambridge University Press.

Corver, N. \& Riemsdijk van, H. 2001. Semi-lexical categories (Studies in Generative Grammar 59). Berlin: Mouton de Gruyter,

Di Sciullo, A-M (1996) Configurations. Somerville, Mass. : Cascadilla Press.

Gosselin, D. (1999) Une analyse en morphologie configurationnelle : le préfixe re- en ancien français. Thèse de doctorat, UQAM.

Hamacher, E. (2002) Le préfixation en français médiéval; une étde du préfixe con-. Thèse de maitrise, Université Queen's.

Klipple, E. (1997). Prepositions and Variation. In Projections and Interface Conditions: Essays on Modularity, Di Sciullo, Anna-Maria (éd.), New York: Oxford U Press, 1997, 74-108.

Marchello-Nizia, C 2002. Prépositions françaises en diachronie : une catégorie en question. Actes du Colloque de Leuven, Adpositions of movement.

Nyrop, K. (1904) Grammaire historique de la langue française. Copenhague : Picard.

Tobler, A.et E. Lommatzsch Altfranzösisches Wörterbuch (complété par H.H. Christmann), Stuttgart: Franz Steiner Verlag, $1915 \mathrm{ff}$.

Victorri B, (1999) Le sens grammatical. Langages, $\mathrm{n}^{\circ}$ 136, vol. décembre. $\mathrm{N}^{\circ}$ spécial "Sémantique lexicale et grammaticale", pp. 85-122.

Wilmet, M. (1998) La Grammaire critique du français. Genève, Droz.

Textes cités de la Base du français médiéval

La Mort le Roi Artu, éd. Jean Frappier, Genève, 1954, Droz (TLF.). 
ISBN 978-2-7598-0358-3, Paris, 2008, Institut de Linguistique Française

Diachronie, histoire de la langue

DOI $10.1051 / \mathrm{cmlf0} 280$

La Queste del Saint Graal, éd. A. Pauphilet, Paris, 1923, Champion (CFMA 33).

La Chanson de Roland, éd. Guy Moignet, Paris, 1970, Bordas.

Tristan en prose (tome 1), éd. Philippe Ménard, Genève, 1987, Droz (TLF 353).

Renaut de Beaujeu : Le Bel Inconnu, éd. G. Perrie Williams, Paris, 1967 (2ème édition), Champion (CFMA 3

\footnotetext{
${ }^{\mathrm{i}}$ Voir Déchaine et Tremblay (en prép.) pour une discussion détaillée du parallélisme entre la distinction comptable/masse qu'on retrouve dans le domaine nominal et la distinction entre les propriétés temporellement variables (comme les événements) et les propriétés temporellement invariables (comme les états).
} 